BMD dual-energy absorptiometry at baseline and at least once thereafter. Two authors independently reviewed references, extracted data and assessed risk of bias. We assessed quality of evidence using the GRADE methodology. Primary outcomes were mean change in BMD at the hip and lumbar spine. Secondary endpoints included RA disease activity and radiographic progression.

Results: We identified 7 studies. Studies were comparable regarding study population and intervention. Risk of bias was considered low for BMD outcomes. Data completeness was low in some studies. We found no statistically significant difference in change in BMD from 0 to 24 months neither at the lumbar spine (Standard Mean Difference (SMD) $0.02(95 \% \mathrm{Cl}-0.16,0.12))$ nor at the hip (SMD $-0.11(95 \% \mathrm{Cl}-0.25,0.02))$. Disease activity was significantly lower in the GC groups (mean difference in DAS28 $-0.32(95 \% \mathrm{Cl}-0.52,-0.11)$. Concomitant treatment of RA differed between studies, as did OP prophylaxis. However, sensitivity analyses excluding a study with different distribution of OP prophylaxis between groups receiving GCs or placebo did not alter the estimates. Quality of evidence was rated moderate for BMD outcomes.

Conclusions: In this group of double-blinded RCT studies we found no difference in change in BMD in patients with RA who received GCs compared to those who received placebo. The interpretation of this is difficult as it challenges the well-established fact that GCs negatively impact BMD. However, our findings suggest that in a population with early RA, followed for two years, the dampening of inflammation as well as increased physical activity may outweigh the inherent effects of GCs. This concurs with our finding of lower disease activity in the groups receiving GCs.

References:

[1] Libanati CR, Baylink DJ. Prevention and treatment of glucocorticoid-induced osteoporosis. A pathogenetic perspective. Chest 1992 Nov;102(5):1426-1435.

[2] van Staa TP, Geusens P, Bijlsma JW, Leufkens HG, Cooper C. Clinical assessment of the long-term risk of fracture in patients with rheumatoid arthritis. Arthritis Rheum 2006 Oct:54(10):3104-3112.

Disclosure of Interest: A.-B. Blavnsfeldt: None declared, M. Thomsen: None declared, S. Tarp: None declared, B. Langdahl Grant/research support from: Novo Nordisk, Eli Lilly and Orkla Health, Speakers bureau: Merck, Amgen, Eli Lilly and UCB, E. Hauge Grant/research support from: AbbVie and Roche, Consultant for: MSD and AbbVie, A. de Thurah: None declared

DOI: 10.1136/annrheumdis-2017-eular.5750

\section{THU0127 THE DYNAMICS OF MENTAL DISORDERS FREQUENCY IN COMPLEX DMARDS, BIOLOGICS AND ANTIDEPRESSANTS TREATMENT OF RHEUMATOID ARTHRITIS PATIENTS}

A. Abramkin ${ }^{1}$, T. Lisitsyna ${ }^{1}$, D. Veltishchev ${ }^{2}$, O. Seravina ${ }^{2}$, O. Kovalevskaya ${ }^{2}$, E. Nasonov ${ }^{1} .{ }^{1}$ Nasonova Research Institute of Rheumatology; ${ }^{2}$ Moscow Research Institute of Psychiatry MoH, Moscow, Russian Federation

Background: mental disorders (MD) (anxiety/depressive (ADD) and cognitive (CD)) occur in rheumatoid arthritis (RA) patients (pts) very often, they are usually stress-related and, probably, have common pathogenesis chains with RA. In this connection the disease-modifying anti-rheumatic drugs (DMARDs) and biologics drugs treatment may be effective in ADD in RA-pts.

Objectives: to determine the frequency of MD dynamics during DMARDs, biologics and antidepressants treatment of RA-pts in prospective 5yrs study.

Methods: 128 RA-pts were enrolled in this study. All of them met the full ACR criteria. $86 \%$ RA-pts were women with a mean age of $47,4 \pm 1,0(\mathrm{M} \pm \mathrm{m}) \mathrm{yrs}$. RA activity was assessed by DAS28 and was high $(5,25 \pm 0,17(\mathrm{M} \pm \mathrm{m}))$ in the beginning of the study. $67 \%$ RA-pts were taking prednisone $(5 \pm 2,7 \mathrm{mg} /$ day), $80 \%$ RA-pts - DMARDs, $26 \%$ - biologics (rituximab - $11 \%$, anti-TNF- $\alpha-10 \%$, anti-IL6 $-5 \%$ ). MD were diagnosed by psychiatrist in accordance with the ICD-10 in semi-structured interview. The severity of depression and anxiety was evaluated by Montgomery-Asberg Depression Rating Scale (MADRS) and Hamilton Anxiety Rating Scale (HAM-A). CD were diagnosed with psychology and neuropsychology methods. ADD were diagnosed in $121(94,5 \%)$ and CD - in 87 (67,9\%) of RA-pts. Major depressive disorder (MDD) was found in $41(32 \%)$, minor depressive disorder (MinDD) - in $50(39 \%)$ and anxiety disorders (AD) - in $30(23,4 \%)$ of RA-pts. The occurrences of MD were evaluated in one and five yrs in 105 from 121 (87\%) RA-pts divided into the following treatment groups: 1 - DMARDS $(n=32)$, 2 - DMARDs + antidepressants (sertraline or mianserine) ( $n=37), 3$ - DMARDs + biologics $(n=27), 4$ - DMARDs + biologics + antidepressants (sertraline or mianserine) $(n=9)$.

Results: Group 1: the frequency of MDD was increased from $24,6 \%$ to $34,8 \%$ in a year and to $42,8 \%$ in 5 yrs $(p=0,09)$; MinDD - from $47,8 \%$ to $60,8 \%(p=0,19)$ and $50 \%$ in 5 yrs; the number of pts with $A D$ decreased from $27,5 \%$ to $4,3 \%$ $(p=0,014)$ and $4,8 \%(p=0,021)$ accordingly. The frequency of $C D$ was increased from $64,3 \%$ to $78,3 \%(p=0,16)$ in 5 yrs. Group 2: the frequency of MDD was decreased from $47,4 \%$ to $15,7 \%(p=0,049)$ in a year and to disappearance of depressive symptoms $(p<0,001)$ in 5 yrs; MinDD - from $26,3 \%$ to $15,8 \%$ and to disappearance $(p<0,001)$ in 5 yrs; $A D$ - from $26,3 \%$ to $10,5 \%$ and to disappearance $(p<0,001)$ in 5 yrs. The frequency of $C D$ was decreased from $78,9 \%$ to $60 \%(p=0,25)$. Group 3: the frequency of MDD was unchanged $(40,7 \%$, $45,4 \%$ and $42,8 \%$ accordingly); the frequency of MinDD was increased not significantly - 40,7\%, 40,9\% and $52,4 \%$; but AD - was significantly decreased (from $18,5 \%$ - to disappearance in a year $(p=0,042)$ and 5 yrs $(p=0,047))$. The frequency of $C D$ was increased from $77,8 \%$ to $85 \%$. Group 4 : the frequency of MDD was decreased significantly from $66,7 \%$ to $16,7 \%$ in a year and to disappearance in 5 yrs $(p=0,03)$; MinDD and AD were decreased from $16,7 \%$ both to disappearance. The occurrence of CD was decreased from $71,4 \%$ to $57,1 \%$. Conclusions: the results demonstrated the best positive dynamics of MD (ADD and $C D$ ) frequency in the groups of RA-pts receiving antidepressants treatment in combination with DMARDs and biologics. There was no positive dynamics of $M D$ in patients group receiving DMARDs and mild positive dynamics of $A D$ in the group receiving biologics.

Disclosure of Interest: None declared

DOI: 10.1136/annrheumdis-2017-eular.3759

\section{THU0128 FEATURES OF ULTRASOUND DOPPLEROGRAPHY IN PATIENTS WITH RHEUMATOID ARTHRITIS AND SUBCLINICAL HYPOTHYROID DYSFUNCTION}

A. Knyazeva ${ }^{1}$, O. Rebrova ${ }^{1}$, N. Bludova ${ }^{1}$, T. Meshcheryakova ${ }^{2}{ }^{1}{ }^{1}$ Internal Medcine, SI "Lugansk State Medical University"; ${ }^{2}$ Ultrasound Department, Lugansk Clinical Regional Hospital, Lugansk, Ukraine

Background: Now it is proved that the leading reason for the decline in life expectancy in patients with rheumatoid arthritis (RA) are cardiovascular (CV) disease. The increase in $\mathrm{CV}$ risk in patients with RA is associated with increased progression of atherosclerotic vascular lesions. Autoimmune inflammatory process in RA affects the vascular endothelium contributes to the appearance of CV events and increased mortality of patients with RA. It is known that when SHTD increased levels of proinflammatory cytokines. Also, when there is an increase SHTD thickness complex intima-media (CIM)

Objectives: To study the characteristics of ultrasound dopplerography in patients with RA with SHTD

Methods: The observation 139 patients with RA. The I group consisted of 91 patients with RA and SHTD, the II - 48 patients with RA without SHTD. Patients in group I and II did not differ significantly by age and duration of RA. Detection of endothelial dysfunction was performed using ultrasound dopplerography vessels in accordance with international guidelines

Results: In the study of endothelial regulation of vascular tone in both groups of patients revealed the presence of disturbances, as determined signs of reduced endothelium-dependent vasodilation (EDVD) and endothelium-independent vasodilation (EIVD) compared with regulatory measures, which EDVD brachial artery (BA) is greater than or equal to $10 \%$ and EIVD BA - 15\%. Defined EDVD significant decrease in group II, in contrast to the I $(T=4.5, p<0.001)$, which amounted to $8.3 \pm 3.0 \%$ and, respectively $10.6 \pm 2.1 \%$. EIVD figure was in group II $12.9 \pm 3.8 \%$, and in the I $-15.4 \pm 1.5 \%$, which also had significant differences $(\mathrm{T}=4.5, \mathrm{p}<0.001)$ (Table 1).

Table 1

\begin{tabular}{lccc}
\hline Indicators & I group $(\mathrm{n}=48)$ & II group $(\mathrm{n}=91)$ & $\mathrm{p}$ \\
\hline $\mathrm{D}_{0}, \mathrm{~cm}$ & $3.8(3.7 ; 3.9)$ & $3.9(3.7 ; 4.2)$ & 0.16 \\
$\mathrm{D}_{1}, \mathrm{~cm}$ & $4.3(4.1 ; 4.5)$ & $4.3(4.0 ; 4.5)$ & 0.83 \\
$\mathrm{D}_{2}, \mathrm{~cm}$ & $4.5(4.3 ; 4.7)$ & $4.5(4.2 ; 4.7)$ & 0.09 \\
$\mathrm{~V}_{0}, \mathrm{~cm} / \mathrm{s}$ & $55.6(52.6 ; 61.1)$ & $52.6(44.2 ; 58.2)^{\star}$ & 0.002 \\
$\mathrm{~V}_{1}, \mathrm{~cm} / \mathrm{s}$ & $76.1(71.6 ; 79.6)$ & $70.8(62.6 ; 76.6)^{\star}$ & $<0.001$ \\
$\mathrm{EDVD}, \%$ & $10.6 \pm 2.1$ & $8.3 \pm 3.0^{\star}$ & $<0.001$ \\
EIVD, \% & $15.4 \pm 1.5$ & $12.9 \pm 3.8^{\star}$ & $<0.001$ \\
$\mathrm{CIM}, \mathrm{mm}$ & $0.73(0.71 ; 0.79)$ & $0.97(0.95 ; 1.0)^{\star}$ & $<0.001$ \\
$\mathrm{C}$ & $0.45(0.34 ; 0.71)$ & $0.26(0.18 ; 0.46)^{\star}$ & 0.002 \\
\hline
\end{tabular}

Note: ${ }^{*} p<0.05$.

It is also a distinction between the two groups in terms of the thickness of the CIM ( $Z=9.7, p<0.001)$, exceeding in group II. Determined a significant decrease in the coefficient of sensitivity to endothelial shear stress $(C)$ in patients with RA, who was in I group of $0.45(0.34 ; 0.71)$, and in II - $0,26(0.18 ; 0.46)$ that there was a statistical difference in this index between the groups of patients $(Z=3.6$, $\mathrm{p}<0.001$ ) due to a significant reduction in patients of group II.

The performed analysis of variance indicated that there SHTD influence on the development of disorders of vascular endothelial function in motor RA patients. The SHTD presence in group II patients significantly affected the reduction EDVD, EIVD, $\mathrm{C}(\mathrm{H}=13.8, \mathrm{p}<0.001 ; \mathrm{H}=14.5, \mathrm{p}<0.001 ; \mathrm{H}=10.2, \mathrm{p}=0.001)$.

Conclusions: The analysis indicated the presence of influence SHTD on the development of disorders of vascular-motor endothelial function in RA patients. These data show that the presence SHTD patients with RA leads to a significant increase in the risk of developing CVD. This requires a more careful study of RA patients for early detection and correction of comorbidities that worsen the clinical course of RA

Disclosure of Interest: None declared

DOI: 10.1136/annrheumdis-2017-eular.3238 